\title{
Influence of shoulder kinematic estimate on joint and muscle mechanics predicted by musculoskeletal model
}

\author{
Y. Blache, M. Begon
}

\begin{abstract}
Goal: Little information is available in the existing literature regarding the influence of the scapular kinematic estimate method on musculoskeletal analysis. This study aimed to assess the propagation of errors due to the method used for scapular kinematics reconstruction in the workflow of musculoskeletal modeling (joint kinematics, joint torques, muscle force and joint reaction force) in shoulder and upper-limb movements. Methods: Two participants performed functional (arm elevation and rotation), daily life (eating and reaching pants pockets) and sports movements (a simulated throwing maneuver). Shoulder kinematics were obtained with five multibody kinematics methods: intra-cortical pins (Pins, reference method), International Society of Biomechanics (ISB), Jackson (Jack), Projection (Proj) and Ellipsoid (Ell) methods. For the five kinematics methods, joint torques, muscle forces and glenohumeral joint reaction forces were computed with the Delft Shoulder and Elbow musculoskeletal model. Results: Differences up to $30^{\circ}$ in glenohumeral joint kinematics, compared to the Pins method, resulted in differences less than $3 \mathrm{~N} . \mathrm{m}$ in joint torque estimation. However, these also resulted in differences up to $50 \mathrm{~N}$ and $831 \mathrm{~N}$ in the muscle force and joint reaction force estimate, respectively, in comparison to the reference method (Pins). No method yielded the worst or best results in comparison to the Pins method but the differences were task-specific. Conclusion: We concluded that shoulder biomechanical studies based on skin markers should be completed with caution when assessing joint angles, muscle forces and glenohumeral joint reaction forces, while researchers may be more confident with the evaluation of shoulder joint torques.
\end{abstract}

Index Terms - soft tissue artefact; muscle force; joint reaction force; joint torque; upper-limb

\section{INTRODUCTION}

M usculoskeletal models are used increasingly more often in the fields of biomechanical engineering [1] and orthopedics [2]. Recordings of skin marker displacements and external forces (e.g., ground reaction) feed the musculoskeletal model to successively estimate joint angles, torques, muscular forces

Copyright (c) 2016 IEEE. Personal use of this material is permitted. However, permission to use this material for any other purposes must be obtained from the IEEE by sending an email to pubs-permissions@ieee.org.

Y. Blache is with the Laboratoire Interuniversitaire de Biologie de la Motricité, Université de Lyon, Lyon, France (e-mail: yoann.blache@univlyon1.fr). M. Begon is with the Laboratoire de Simulation et Modélisation du Mouvement, Département de Kinésiologie, Université de Montréal, Québec, Canada, (e-mails: mickaelbegon@gmail.com). and joint reaction forces [3]. However, soft tissue artifacts (STA), which correspond to the movement of a skin marker relative to its underlying bone [4], can affect joint kinematics and propagate thereafter. In terms of the upper limbs, Matsui, Shimada and Andrew [5] observed STA up to $8 \mathrm{~cm}$ of skin markers located on the scapula. Blache, Dumas, Lundberg and Begon [6] pointed out that STA of the upper limbs and shoulders were mainly characterized by rigid transformations. Finally, errors up to $30^{\circ}$ on the arm axial rotation were found when using an electromagnetic sensor attached to the lateral aspect of the arm [7].

To overcome inaccuracy caused by STA of the upper limbs, multibody kinematics optimization is used [8]. It consists of minimizing the quadratic distance between the position of the experimental skin markers and the estimated position of markers located on a kinematic chain model. Several marker sets and various constraints were tested to improve scapular and humeral kinematics in particular. As a first step, the International Society of Biomechanics [9] has suggested using sets of two to four markers per segment to estimate upper limb bone kinematics. Increasing the number of markers on each segment may also improve kinematic accuracy [10]. The scapulo-thoracic pseudo-joint has also been implemented as a contact with an ellipsoid fitted to the semi-thorax to enforce a curvature displacement of the scapula throughout the movement [11-13]. Finally, projection methods, which consist of neglecting the effect of a given marker on one degree of freedom, can possibly improve the accuracy of shoulder kinematic estimates [14, 15]. In consequence, it is commonly accepted that the method used during multibody kinematics optimization affects the calculation of shoulder joint angles [8, 13, 15].

However, little information is available in the literature about the upper limb STA-propagated effects on joint torques, muscle forces and joint reaction force estimates. Only $\mathrm{Wu}$ et al. [16] have used a Monte Carlo analysis to assess the effect of kinematic changes on muscle and joint mechanics. They concluded that the sensibility of shoulder muscle forces to joint kinematics was muscle and task specific. However, in this study [16], only arm elevations in the sagittal and coronal planes were presented. In addition, kinematic changes were simulated from a baseline that was not free of error since the kinematics were measured with skin markers. Therefore, it would be relevant, firstly, to test a broader range of movements and secondly, to compare joint and muscle 
mechanics with data obtained from kinematics which are free from errors in order to confirm previous conclusions.

The purpose of this study was to assess the propagation of errors caused by the method used for scapular kinematic reconstruction in the workflow of musculoskeletal modeling during analytical, sports-related and daily life movements. It was hypothesized that kinematic errors affect muscle forces, joint reaction forces and, to a lesser extent, joint torques.

\section{METHODS}

\section{A. Participants}

The raw data presented by Dal Maso, Raison, Lundberg, Arndt and Begon [17] were used for the current study. Two healthy male participants (P1: 44 years; $1.77 \mathrm{~m}$; $82 \mathrm{~kg}$ and P2: 27 years; $1.65 \mathrm{~m} ; 57 \mathrm{~kg}$ ) took part in this study. They signed an informed consent document, which was approved by the ethics committees at the Karolinska Institute (Sweden) and the University of Montreal (Canada). None of the participants had previous or current shoulder injuries.

\section{B. Instrumentation}

After administration of local anesthesia (AstraZeneca, Södertälje, Sweden), four to five reflective markers secured to pins were inserted into each of the following areas: below the attachment of the medial deltoid on the humerus, into the first third of the scapula spine and into the lateral part of the clavicle. See Dal Maso, Raison, Lundberg, Arndt and Begon [17] for details about the surgical insertion. In addition, 32 skin markers were placed on the thorax (4), left clavicle (6), scapula (8), upper arm (7), lower arm (3) and hand (4). Marker trajectories of the movements were measured using an 18camera optoelectronic VICON ${ }^{\mathrm{TM}}$ system (Oxford Metrics Ltd., Oxford, UK, $300 \mathrm{~Hz}$ ).

\section{Experimental procedures}

Prior to the test, the participants held an upright static standing position for five seconds with their arms alongside their bodies for calibrating the model. The first series of movements of interest consisted of three different analytical movements, which were performed up to 11 times each. These included raising and lowering the arms in both the sagittal (flexion/extension) and the frontal planes (abduction /adduction), and internal arm rotations with $0^{\circ}$ of arm abduction. For the arm raising and lowering movements, participants had to maintain a neutral internal arm rotation and keep the elbow extended throughout the movements. For the arm rotations, participants held their elbows at $90^{\circ}$ of flexion throughout the movement. In a second series, the participants performed six repetitions of three daily-life activities (i.e., mimicking eating, reaching their back, and front pants pockets) and a sport movement (i.e., a simulated throwing maneuver with one bent arm). The participants performed their movements at their preferred speed. Among all of the repetitions of each movement, the three repetitions performed with the most similar speed were used for the analysis.

\section{Musculoskeletal model}

We used the Delft Shoulder and Elbow model [18], which is available with OpenSim [3]. This model is composed of eight segment: ground, thorax, left clavicle, scapula, humerus, ulna, radius and hand. The segments were inter-connected with 19 degrees of freedom, namely the ground-thorax joint (3 translations and 3 rotations), sterno-clavicular joint (3 rotations), acromio-clavicular joint (3 rotations), glenohumeral joint ( 3 rotations), elbow ( 2 rotations) and wrist ( 2 rotations). The model was actuated by 139 muscle lines of action and 19 residual actuators (i.e., artificial joint actuators used to help the model when muscles could not produce the joint torque required to perform the movement). Twelve ligaments provided additional passive elastic forces. The uniform OpenSim 3D-scaled function was implemented to scale the generic model to each participant's anthropometry. To that aim, the skin marker data obtained from the static poses, which were not subject to STA in this condition, were used (Spinous Process of the $7^{\text {th }}$ cervical and $8^{\text {th }}$ thoracic vertebra, Incisura Jugularis and Processus Xiphoideus for the thorax; Acromioclavicular and Sternoclavicular joints for the clavicle; Trigonum Scapulae, Angulus Inferior and Acromialis for the scapula; Medial, Lateral Epicondyles and glenohumeral joint determined by regression equation, for the humerus [19]; Radial, Ulnar Styloids and Olecranon for the forearm; distal and proximal parts of the $2^{\text {nd }}$ and $4^{\text {th }}$ Metacarpus for the hand).

\section{E. Inverse kinematics}

Five methods were used to compute joint angles (Fig. 1). For each method, the same multibody kinematics optimization algorithm was implemented, which entailed minimizing the quadratic weighted Euclidean distance between the experimental markers and the virtual markers of the kinematic model.

In the first method (Pins), joint angles were obtained by considering the skin markers located on the thorax, lower arm and hand, and the pin markers of the clavicle, scapula and humerus. To avoid the negative effect of soft tissue artefact caused by the skin markers, high (20), medium (10) and low (1) weightings were allocated to the markers put on the pins, thorax and "forearm + hand", respectively. For the other four methods, only skin markers were used, and the methods differed only at the scapula segment. The second method (ISB) was based on the recommendation of the International Society of Biomechanics [9], which suggested four markers on the scapula: inferior angle, Trigonum scapulae, Coracoideus process and angulus acromialis. For the third method (Jack), the marker set used by Jackson, Michaud, Tetreault and Begon [10] was implemented (Coracoideus process, acromion tip, acromion angle, lateral and middle part of the scapular spine). The fourth method (Proj) was based on the study of Naaim, Moissenet, Dumas, Begon and Cheze [14]. The inferior angle marker was added to the Jack marker set and projected onto the $\mathrm{z}$-axis of the scapula. The projected marker has thus no effect on the upward-downward rotation axis. In the fifth method (Ell), the same marker set from the Jack method was applied, and an ellipsoid fitted to the semi-left-thorax was implemented with a two-point-on-ellipsoid constraint. The marker residuals were reported as recommended by Begon, Andersen and Dumas [20]. 


\section{F. Joint and muscle mechanics}

Firstly, joint angles were filtered using a zero-lag fourth-order low-pass Butterworth filter with a $6 \mathrm{~Hz}$ cut-off frequency. Then, OpenSim 3.2 [3] was used to compute the joint torques from the joint kinematics which were obtained with the five methods. Thereafter, muscle forces were estimated for each sample time with a constrained static optimization algorithm. Briefly, the objective function was to determine the leastsquared muscle activations and residual torques (reported in the results section), such that the joint torques due to muscle forces were equal to those calculated by inverse dynamics. An additional constraint to ensure glenohumeral joint stability was implemented by linear approximation of a revolution cone that determine friction limits of normal and tangential forces according to the Coulomb's law [21, 22]. The sternoclavicular, acromioclavicular and glenohumeral contact forces were computed using the jointReaction algorithm of OpenSim 3.2 $[3,23]$.
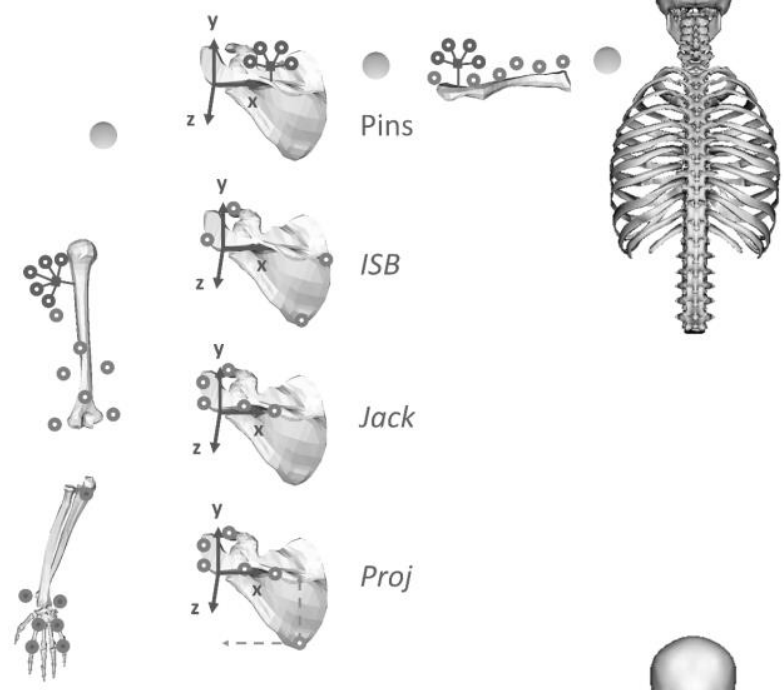

Jack

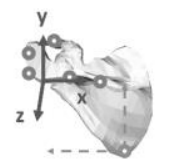

Proj
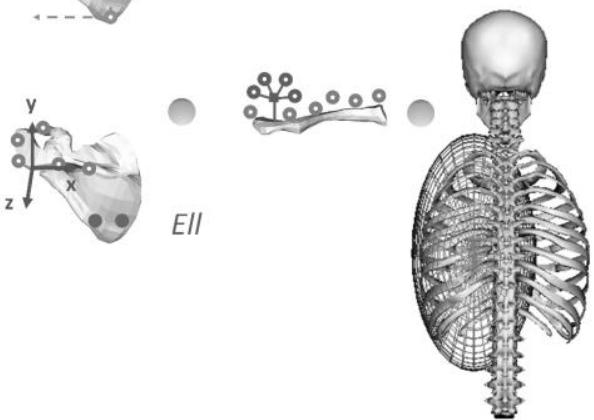

Fig. 1. Presentation of the marker set used for the five methods: Pins (reference), ISB (International Society of Biomechanics [9] marker set), Jack (Jackson, Michaud, Tetreault and Begon [10] marker set), Proj (inferior angulus marker with no effect on the upward-downward rotation axis [14]) and Ell (ellipsoid fitted to the semi-left-thorax [12]).

\section{G. Evaluation of the model outputs}

The kinematic model outputs were assessed by computing the average and maximal marker residuals. Mean and maximal residual torques were computed to evaluate the capacity of the muscles to perform the given movements. Finally, estimated glenohumeral joint reaction forces were compared with invivo data provided by the literature [24-26].

\section{H. Analysis}

Since only two participants were involved, descriptive statistics were provided for joint kinematics, joint torques, muscle forces and joint reaction forces. The root mean square difference (RMSd) between the Pins time histories (reference method for the joint kinematics) and the other four methods (ISB, Jack, Proj and Ell) was calculated for joint kinematics, joint torques, muscle forces and joint reaction forces.

\section{RESULTS}

\section{A. Evaluation of the Model outputs}

The average marker residuals ranged from 5 to $14 \mathrm{~mm}, 8$ to $23 \mathrm{~mm}, 7$ to $23 \mathrm{~mm}, 13$ to $35 \mathrm{~mm}$ and 15 to $38 \mathrm{~mm}$ for the Pins, ISB, Jack, Proj and Ell methods, respectively. The maximal marker residuals were observed for the Proj and Ell methods (between 27 and $123 \mathrm{~mm}$ ) (see supplementary file 'supp_file1.xlsx' for more details). In addition, for the Pins method, the maximal marker residuals were never observed for the pin markers, and instead were mostly seen for the skin markers located on the hand and wrist.

On average, mean residual torques were found to be between 0.05 and 0.45 N.m, and maximal residual torques ranged from 0.25 to 1.46 N.m. This was on average 41,21 and 73 times smaller than the corresponding maximal joint torques for the sternoclavicular, acromioclavicular and glenohumeral joints respectively. Finally, regardless of the movement and method, no saturation of muscle activation was observed.

\section{B. Joint kinematics}

The RMSd of the sternoclavicular joint kinematic between the Pins method and the other methods varied from 0.29 to $11.10^{\circ}$, regardless of the participant (see supplementary file 'supp_file2.xlsx' for more details). The greatest differences were observed with the ISB, Proj and Ell methods, and in descending order for eating, abduction and adduction, flexion and extension, simulated throwing maneuver, pocket reaching and arm rotation movements. For the acromioclavicular joint, RMSd ranged between 0.40 and $11.75^{\circ}$. The greatest differences were primarily observed with the Jack method, and in descending order for flexion and extension, abduction and adduction, pocket reaching, eating, simulated throwing maneuver and arm rotation movements. For the glenohumeral joint, the RMSd were between 1.05 and $33.32^{\circ}$. The greatest differences alternated among all of the methods and were observed in descending order for flexion and extension, eating, simulated throwing maneuver, arm rotation, pocket reaching, and abduction and adduction movements. Finally, for the scapulothoracic joint, RMSd ranged from 0.45 to $18.11^{\circ}$. The greatest differences were mostly observed with the Jack method and were observed in descending order for flexion and extension, eating, simulated throwing maneuver, arm rotation movements, pocket reaching and abduction (Fig. 2, Fig. Supp1 and Fig. Supp2). 


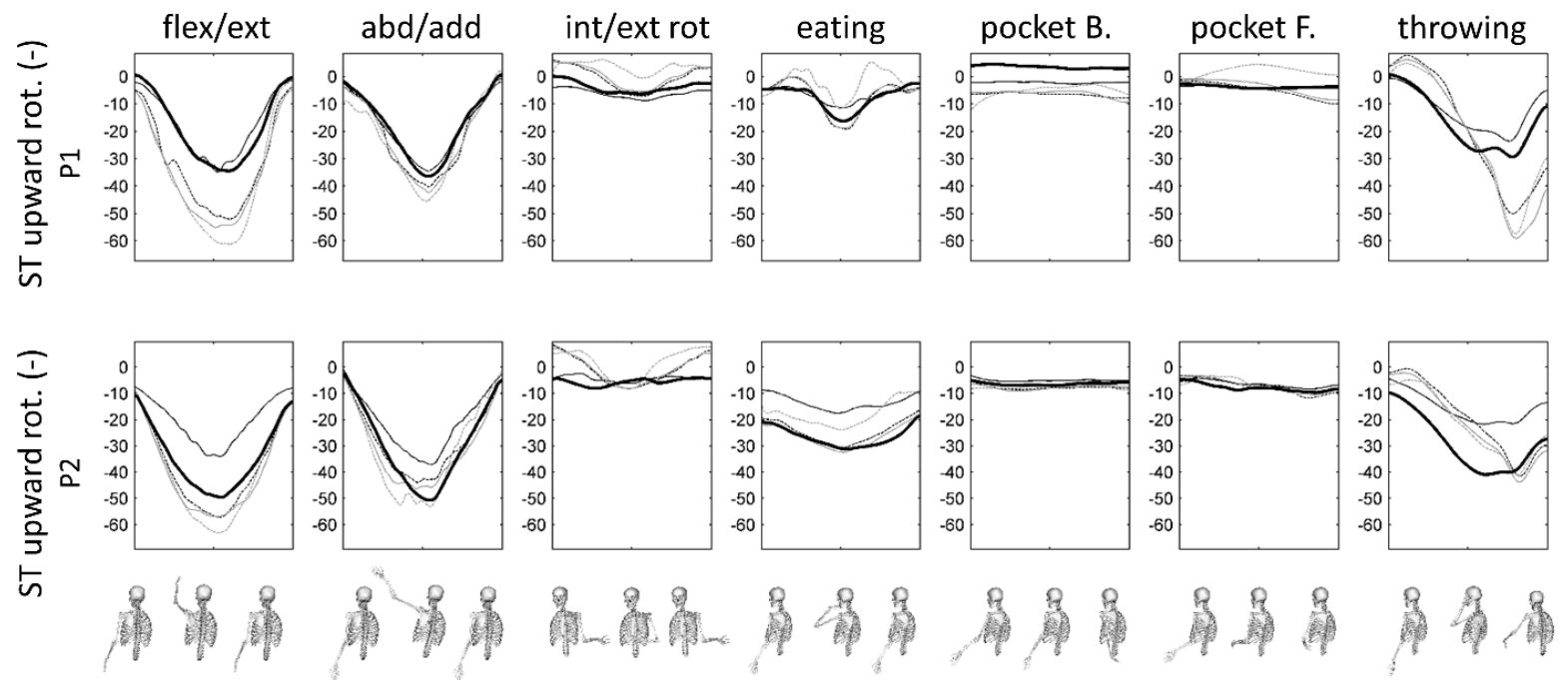

Fig. 2. Scapulothoracic (ST) upward/downward rotation angles in degrees during the seven movements (flexion/extension (flex/ext), abduction/adduction (abd/add), internal/external rotation (int/ext rot), eating, reaching back (pocket B.) and front (pocket F.) pockets, simulated throwing maneuver) with respect to the normalized time (between 0 and 1) for the two participants (P1 and P2). The five methods are represented: Pins (dark thick solid line), International Society of Biomechanics (dark thin solid line), Jackson et al. (2012) (dark dashed line), Projection method (grey thin solid line) and Ellipsoid method (grey dashed line).

\section{Joint torques}

Regardless of the movement and participant, the maximal RMSd of the joint torques were 0.87 N.m, 1.96 N.m and 2.64 N.m for the sternoclavicular, acromioclavicular and glenohumeral joints respectively (see supplementary file 'supp_file2.xlsx' for more details). For the sternoclavicular joint, RMSd was always inferior to $10 \%$ of the peak joint torque, no matter the movement. Concerning the acromioclavicular joint, maximal RMSd corresponded to $24 \%$, $45 \%, 6 \%, 16 \%, 26 \%, 13 \%$ and $25 \%$ of the peak joint torque for flexion and extension, abduction and adduction, arm
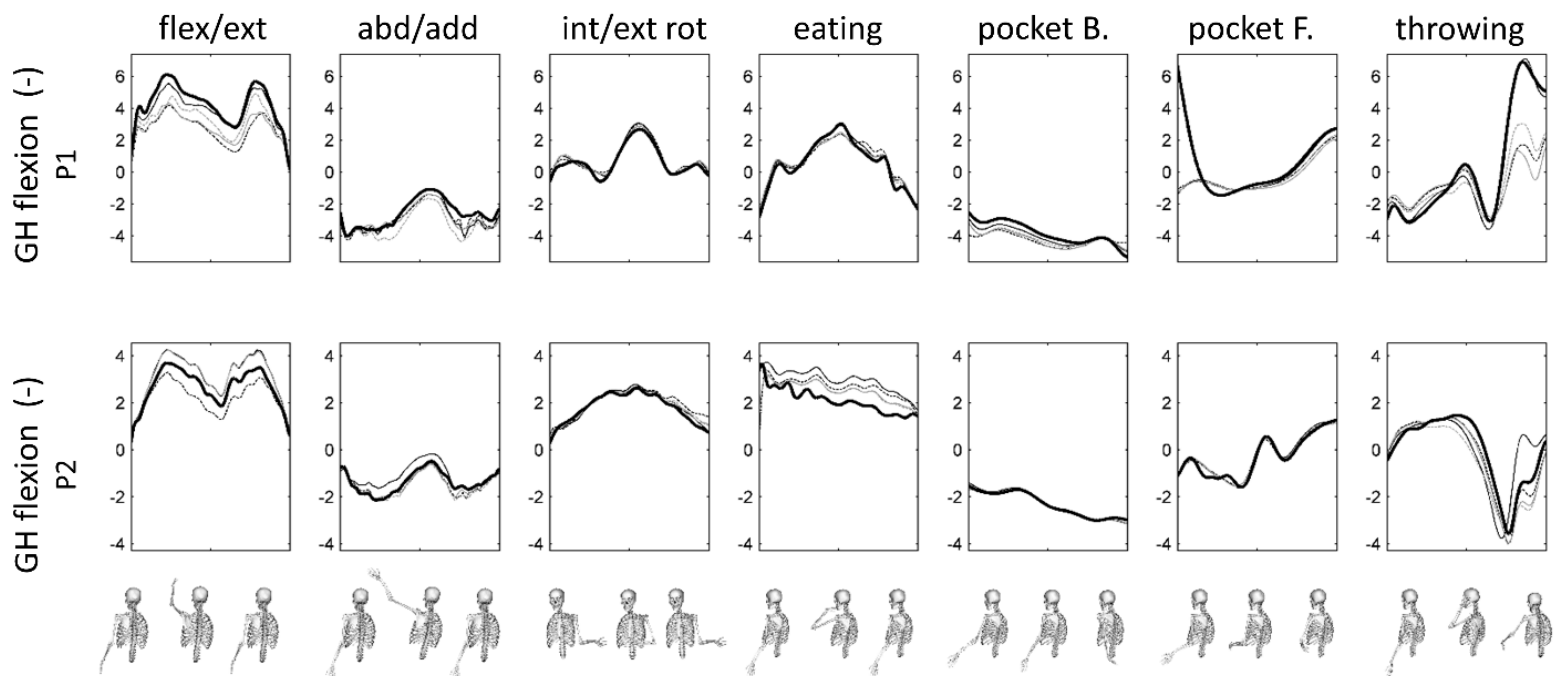

Fig.3: Glenohumeral (GH) flexion/extension torque in newton during the seven movements (flexion/extension (flex/ext), abduction/adduction (abd/add), internal/external rotation (int/ext rot), eating, reaching back (pocket B.) and front (pocket F.) pockets, simulated throwing maneuver) with respect to the normalized time (between 0 and 1) for the two participants (P1 and P2). The five methods are represented: Pins (dark thick solid line), International Society of Biomechanics (dark thin solid line), Jackson et al. (2012) (dark dashed line), Projection method (grey thin solid line) and Ellipsoid method (grey dashed line). 


\section{Muscle forces}

Irrespective of the movement and the participant, the maximal RMSd of the muscle were 11, 50, 6, 25, 47, 28 and $29 \mathrm{~N}$ for flexion and extension, abduction and adduction, arm rotation, eating, reaching back and front pockets and simulated throwing maneuver, respectively (see supplementary file 'supp_file2.xlsx' for more details). Among the 22 muscle groups, the 5 muscle groups producing the greatest force were the upper trapezius, anterior deltoid, middle deltoid, infraspinatus and subscapularis. For these muscles, the maximal RMSd ranged from $6 \%$ to $34 \%, 7 \%$ to $70 \%, 9 \%$ to $28 \%, 8 \%$ to $56 \%$ and $7 \%$ to $58 \%$ of the peak muscle force for the upper trapezius, anterior deltoid, middle deltoid, infraspinatus and subscapularis, respectively. The movements of reaching for the back and front pockets presented the greatest RMSd, while no method consistently yielded the highest or lowest RMSd (Fig. 4-5 and Fig. Supp11 to Fig. Supp13).
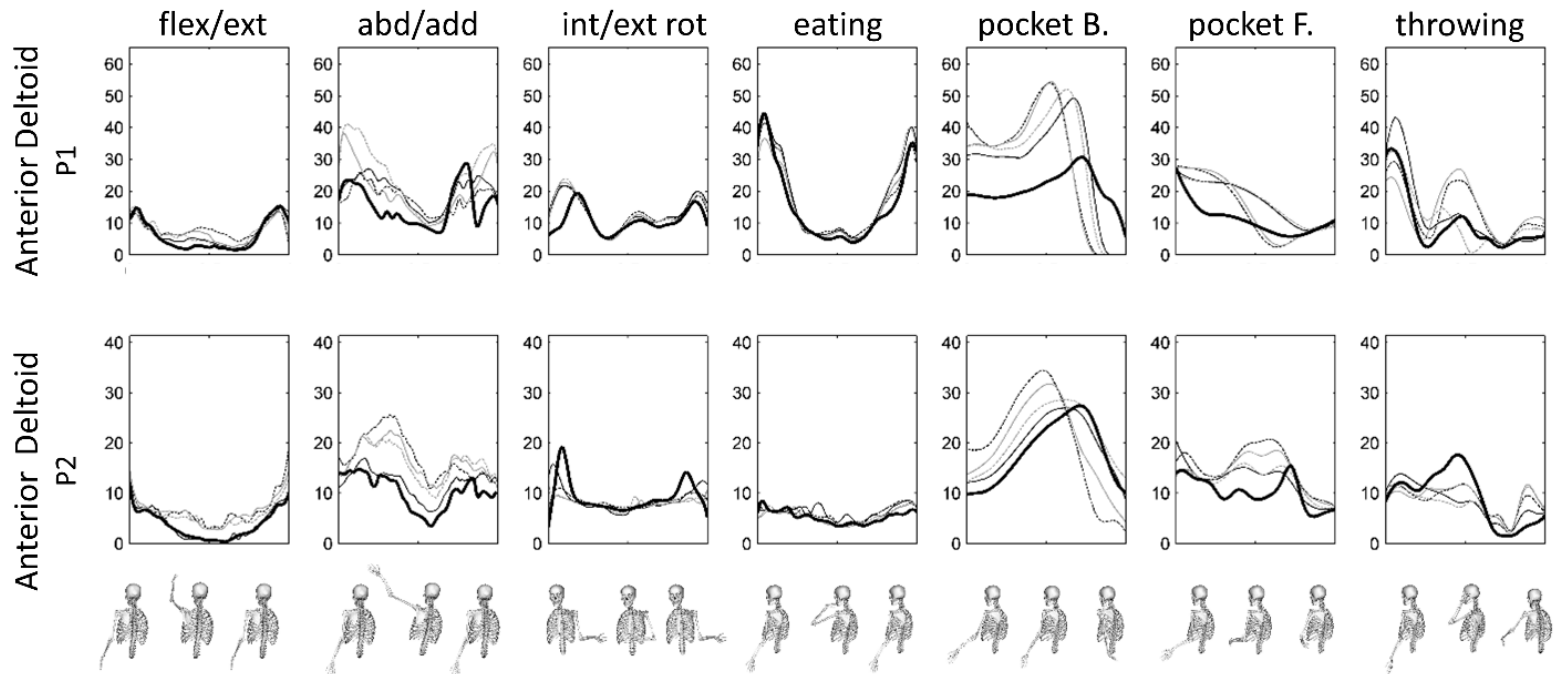

Fig 4: Anterior deltoid estimated force expressed in newton during the seven movements (flexion/extension (flex/ext), abduction/adduction (abd/add), internal/external rotation (int/ext rot), eating, reaching back (pocket B.) and front (pocket F.) pockets, simulated throwing maneuver) with respect to the normalized time (between 0 and 1) for the two participants (P1 and P2). The five methods are represented: Pins (dark thick solid line), International Society of Biomechanics (dark thin solid line), Jackson et al. (2012) (dark dashed line), Projection method (grey thin solid line) and Ellipsoid method (grey dashed line).

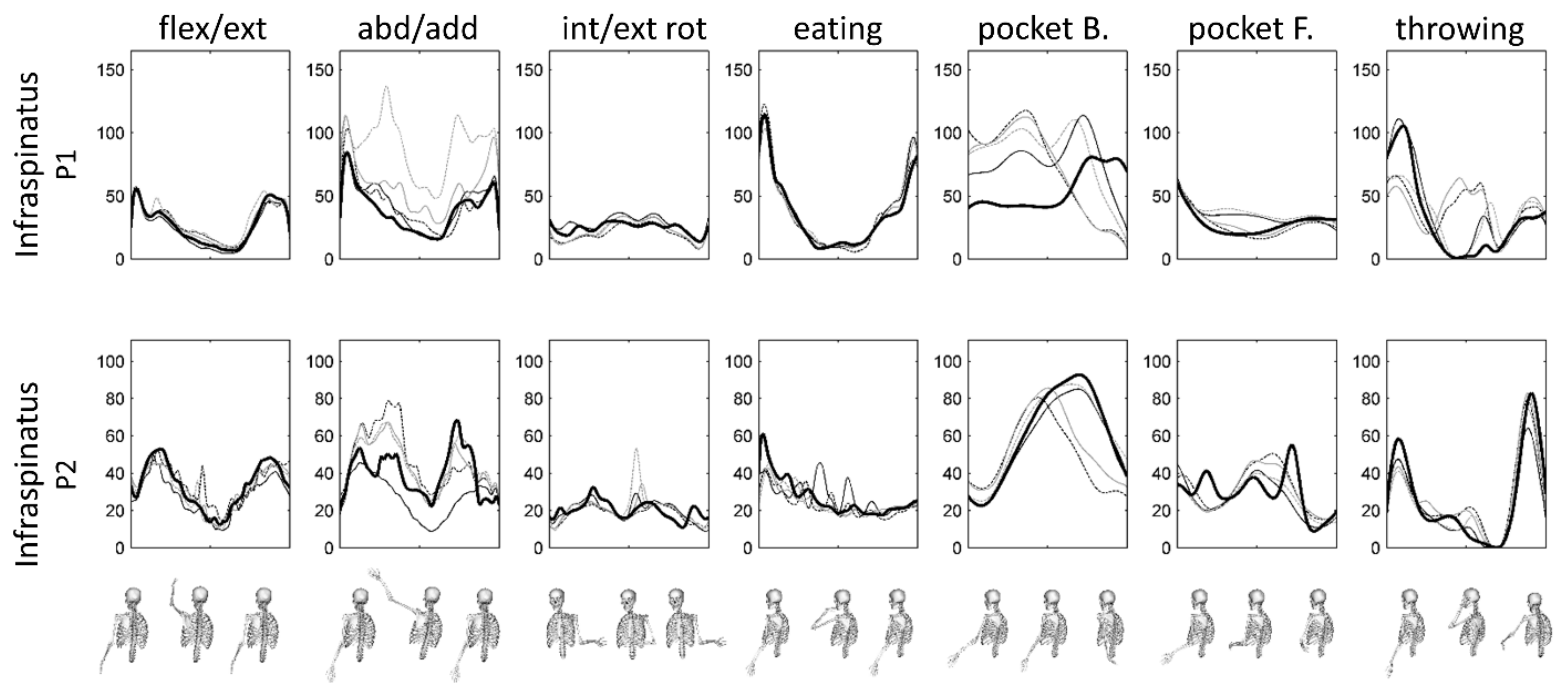

Fig 5: Infraspinatus estimated force expressed in newton during the seven movements (flexion/extension (flex/ext), abduction/adduction (abd/add), internal/external rotation (int/ext rot), eating, reaching back (pocket B.) and front (pocket F.) pockets, simulated throwing maneuver) with respect to the normalized time (between 0 and 1) for the two participants (P1 and P2). The five methods are represented: Pins (dark thick solid line), International Society of Biomechanics (dark thin solid line), Jackson et al. (2012) (dark dashed line), Projection method (grey thin solid line) and Ellipsoid method (grey dashed line). 


\section{E. Joint reaction force}

The RMSd of the sternoclavicular, acromioclavicular and glenohumeral joint reaction forces between the Pins and the other methods were from 6 to $197 \mathrm{~N}, 3$ to $139 \mathrm{~N}$ and 32 to $831 \mathrm{~N}$, respectively (see the supplementary file for more details). Maximal RMSd corresponded to $10 \%, 68 \%, 15 \%$,
$6 \%, 64 \%, 49 \%$ and $29 \%$ of the peak glenohumeral joint reaction force for flexion and extension, abduction and adduction, arm rotation, eating, reaching back and front pockets and simulated throwing maneuver, respectively. The highest RMSd alternated among the four methods (Fig. 6).

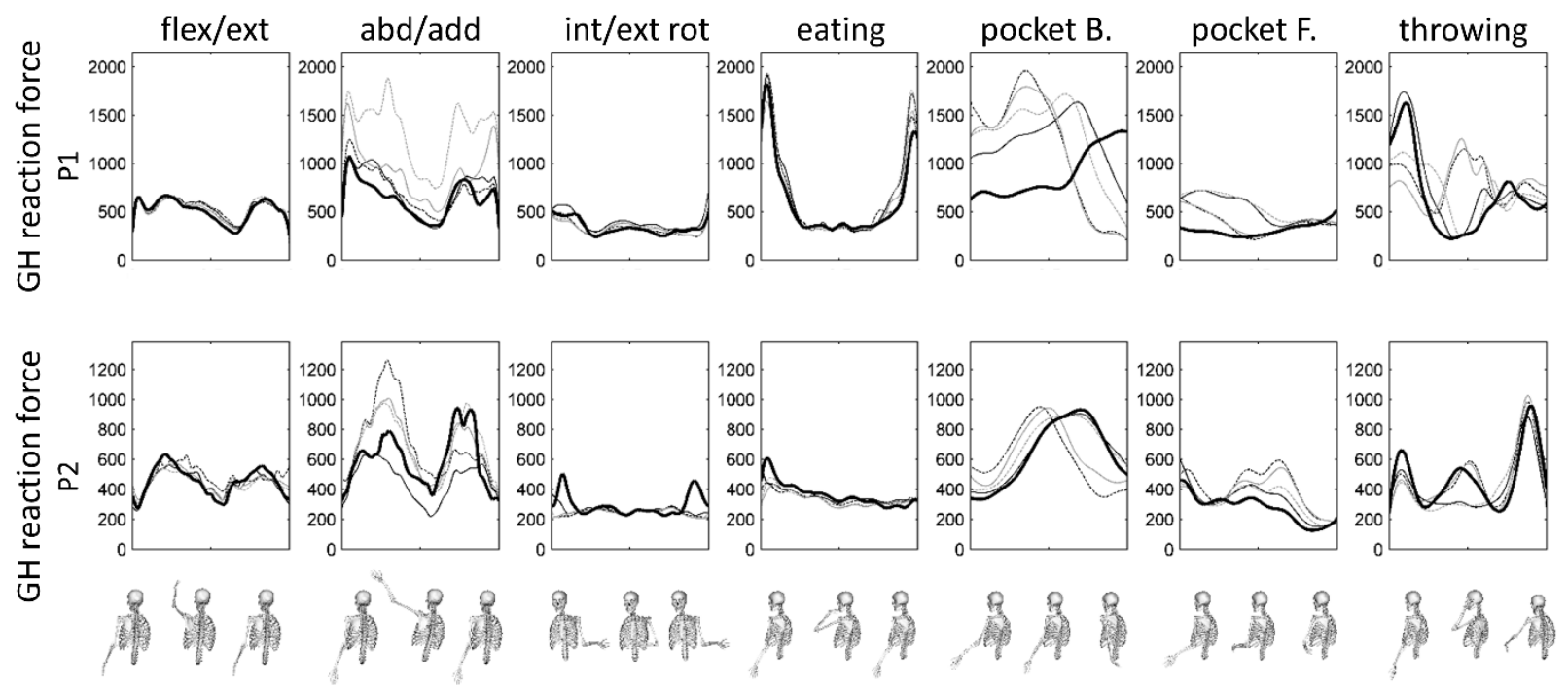

Fig 6: Glenohumeral (GH) joint reaction force in Newton during the seven movements (flexion/extension (flex/ext), abduction/adduction (abd/add), internal/external rotation (int/ext rot), eating, reaching back (pocket B.) and front (pocket F.) pockets, simulated throwing maneuver) with respect to the normalized time (between 0 and 1) for the first participant (P1 and P2). The five methods are represented: Pins (dark thick solid line), International Society of Biomechanics (dark thin solid line), Jackson et al. (2012) (dark dashed line), Projection method (grey thin solid line) and Ellipsoid method (grey dashed line).

\section{DISCUSSION}

The purpose of this study was to assess the propagation of errors caused by the method used for scapular kinematic reconstruction in the workflow of musculoskeletal modeling. We placed emphasis on the scapula model during analytical, sports-related and daily life movements. The main finding was that, in comparison to the Pins method, glenohumeral kinematic differences up to $30^{\circ}$ resulted in differences inferior to 3 N.m in joint torque estimation. However, this also resulted in differences up to $50 \mathrm{~N}$ and $831 \mathrm{~N}$ for the muscle force and joint reaction force estimates, respectively.

\section{A. Evaluation of the model outputs}

Marker residuals were similar to those observed by previous studies [27, 28]. In addition, as recommended by Begon, Andersen and Dumas [20], marker residuals were in the same range as values of STA observed for the shoulder and upperlimbs [5]. Consequently, despite the multibody kinematics optimization method, no numerical problems were put forward when computing joint angles.

Regardless of the movement and the method, the mean and maximal residual torques were negligible. The absence of the muscle activation saturation and the residual torques values which were in agreement with the recommendations of Hicks, Uchida, Seth, Rajagopal and Delp [29], meant that, no matter the method and movement, the muscles of the musculoskeletal model were able to produce the required forces. Therefore, the
Delft Shoulder and Elbow model [18] is sufficiently actuated for the movements which were analyzed in this study.

Although only indirect comparison with the literature is possible, glenohumeral ligaments seemed to well reproduce in-vivo data. Especially, we observed similar glenohumeral ligament elongations between our simulated ball throwing task and humeral internal-external rotations [30] (between 51 and $106 \%$ on average vs. between 40 and $130 \%$ for Massimini, Boyer, Papannagari, Gill, Warner and Li [30]).

The glenohumeral joint reaction forces estimated with the Pins method during flexion and abduction movements were slightly higher than those observed in-vivo [24]. We had maximal glenohumeral forces equal to $92 \%$ and $143 \%$ of body weight in the present study for flexion and abduction movements respectively. Values of $90 \%$ and $120 \%$ of body weight were observed in six patients fitted with instrumented implants [24]. Nevertheless, the orders of magnitude were the same, and we also found greater glenohumeral joint reaction force during the abduction/adduction than during the flexion/extension movements. Several parameters of the musculoskeletal model affect joint reaction force estimates and possibly explain the differences between our model and in-vivo data. Such parameters include the muscle characteristics, scaling method, stability constraint and cost function [25, 26]. Unfortunately, no in-vivo data are available in the literature for the comparison of the other movements. In summary, we found that the musculoskeletal model respected the salient 
characteristics of the analyzed movements, enabling the interpretation of the results.

\section{B. Muscle and joint mechanical outputs}

Our results confirm previous findings [8] that STA of the upper limbs affect joint angle estimates, although several recent approaches have been implemented to decrease this negative effect. Even though high kinematic differences with the Pins method (up to $33^{\circ}$ for the glenohumeral joint) were observed, as in the studies of Ludewig' group (e.g. Hamming, Braman, Phadke, LaPrade and Ludewig [7]) and our group (e.g., Begon, Belaise, Naaim, Lundberg and Cheze [15]), only small differences of about $3 \mathrm{~N} . \mathrm{m}$ on joint torques were noticed. While the joint angles differed among the methods, we assume that their accelerations were slightly affected. These results are in accordance with previous studies which observed small effects of the STA on net joint torque estimates of the lower limbs [31].

By contrast, muscle force estimates are sometimes drastically affected by STA [16, 31]. Indeed, when computing muscle forces, a kinematic change of just a few degrees changes the muscle moment arm and, consequently, the muscle force required to produce the appropriate joint torque. As observed for the lower limbs [31, 32] and recently for the upper limbs [16], we pointed out that the maximal RMSd reached more than $50 \%$ of the peak muscle force. The infraspinatus, subscapularis (rotator cuff muscles) and anterior deltoid presented the greatest RMSd in comparison to the Pins method. According to $\mathrm{Wu}$, Lee and Ackland [16], the muscle force RMSd was task specific. Firstly, for a given task, the muscles that produced the greatest forces tended to exhibit the greatest RMSd. Secondly, on average, the greatest RMSd was observed with the pocket-reaching and simulated throwing tasks. Similarly to Wu, Lee and Ackland [16] smaller RMSd were observed for flexion in comparison to abduction.

According to $\mathrm{Wu}$, Lee and Ackland [16] glenohumeral joint reaction forces were affected by STA. When compared to the Pins method, we also observed a significant overestimation of the glenohumeral joint reaction force (RMSd up to $64 \%$ of peak value) with the other methods. This can probably be explained by the addition of the differences observed for net joint torques and muscle forces, since joint reaction force depends on these two parameters [23]. We also noticed that joint reaction forces RMSd were task specific [16], with the greatest differences observed with the pocket-reaching and abduction/adduction tasks. Finally, the effect of the method used during the multibody kinematics optimization on joint and muscle mechanics is not straightforward. Indeed, no method always yielded the closest or farthest result in comparison to the Pins method.

The propagation of STA error in musculoskeletal analysis highlights the importance of the precautionary principle when interpreting data in the fields of biomedical engineering [3335], orthopedics [36] or sports biomechanics [22]. Unsurprisingly, shoulder biomechanical studies based on skin markers or sensors should be considered with caution since major differences were observed in comparison to Pins methods [7]. By contrast, researchers might be more confident with the values of net joint torques of the upper limb regardless of the multibody kinematics optimization methods.
In musculoskeletal studies that estimate muscle force and glenohumeral joint reaction forces, some cautions must be taken since STA led to great differences, in comparison to the Pins method. Nevertheless, since the shape of the curves of the five methods were mostly similar, we advise to discuss more about the tendencies than the absolute values of the muscle and glenohumeral joint reactions forces.

\section{Limitations}

The first limitation is the small number of participants enrolled in this study. Further studies using fluoroscopy or intra-cortical pins with more participants should be conducted. The second limitation was that only one musculoskeletal model was tested, which limited the extrapolation of our results to other shoulder musculoskeletal models. Nevertheless, the Delft Shoulder and Elbow model has been shown to be suitable for reproducing and simulating human movements $[25,26]$. The other models present some drawbacks, such as the small number of muscles [37] or the limited number of degrees of freedom [38]. The third limitation was that several parameters of the musculoskeletal model, such as the muscle-tendon characteristics and the cost function may also influence muscle force and glenohumeral contact force estimates. For these reasons, more sensitivity studies are needed to address the effect of these parameters.

\section{CONCLUSION}

This study presents useful highlights about the propagation of errors due to shoulder kinematic estimate method in the workflow of musculoskeletal modeling. Although changes in kinematics estimates were observed, the assessment of shoulder joints torques appears relatively accurate. However, when estimating muscle forces and glenohumeral joint reaction forces, trends should be discussed rather than absolute values. Consequently, further studies are still needed to improve kinematic estimates of the shoulder joint in order to improve the accuracy of the musculoskeletal outputs.

\section{DISCLOSURE}

The work presented in this paper has neither financial support nor author- or project-related conflict of interest.

\section{REFERENCES}

[1] B. Bolsterlee, D. H. Veeger, and E. K. Chadwick, (2013, Sep.). Clinical applications of musculoskeletal modelling for the shoulder and upper limb. Med Biol Eng Comput. 51(9), pp. 953-963.

[2] W. Liou, Y. Yang, G. R. Petersen-Fitts, D. J. Lombardo, S. Stine, and V. J. Sabesan, (2017, Apr.). Effect of lateralized design on muscle and joint reaction forces for reverse shoulder arthroplasty. $J$ Shoulder Elbow Surg. 26(4), pp. 564-572.

[3] S. L. Delp, F. C. Anderson, A. S. Arnold, P. Loan, A. Habib, C. T. John, E. Guendelman, and D. G. Thelen, (2007, Nov.). OpenSim: open-source software to create and analyze dynamic simulations of movement. IEEE Trans Biomed Eng. 54(11), pp. 1940-1950.

[4] A. Cereatti, T. Bonci, M. Akbarshahi, K. Aminian, A. Barre, M. Begon, D. L. Benoit, C. Charbonnier, F. Dal Maso, S. Fantozzi, C. C. Lin, T. W. Lu, M. G. Pandy, R. Stagni, A. J. van den Bogert, and V. Camomilla, (2017, Feb.). Standardization proposal of soft tissue artefact description for data sharing in human motion measurements. $J$ Biomech. 
[5] K. Matsui, K. Shimada, and P. D. Andrew, (2006, Mar.). Deviation of skin marker from bone target during movement of the scapula. J Orthop Sci. 11(2), pp. 180-184.

[6] Y. Blache, R. Dumas, A. Lundberg, and M. Begon, (In Press, Oct.). Main component of soft tissue artifact of the upper-limbs with respect to different functional, daily life and sports movements. $J$ Biomech.

[7] D. Hamming, J. P. Braman, V. Phadke, R. F. LaPrade, and P. M. Ludewig, (2012, Apr.). The accuracy of measuring glenohumeral motion with a surface humeral cuff. J Biomech. 45(7), pp. 11611168.

[8] S. Duprey, A. Naaim, F. Moissenet, M. Begon, and L. Cheze, (In Press, Dec.). Kinematic models of the upper limb joints for multibody kinematics optimisation: An overview. J Biomech.

[9] G. Wu, F. C. van der Helm, H. E. Veeger, M. Makhsous, P. Van Roy, C. Anglin, J. Nagels, A. R. Karduna, K. McQuade, X. Wang, F. W. Werner, B. Buchholz, and B. International Society of, (2005, May.). ISB recommendation on definitions of joint coordinate systems of various joints for the reporting of human joint motion-Part II: shoulder, elbow, wrist and hand. J Biomech. 38(5), pp. 981992.

[10] M. Jackson, B. Michaud, P. Tetreault, and M. Begon, (2012, Aug.). Improvements in measuring shoulder joint kinematics. $J$ Biomech. 45(12), pp. 2180-2183.

[11] A. Seth, R. Matias, A. P. Veloso, and S. L. Delp, (2016, A Biomechanical Model of the Scapulothoracic Joint to Accurately Capture Scapular Kinematics during Shoulder Movements. PLoS One. 11(1), pp. e0141028.

[12] B. Michaud, and B. Begon, "Thorax ellipsoid optimization based on scapula movements' area improves kinematic reconstruction of the scapula," in Digital Human Modeling Congress, Montreal Canada, 2016, July.

[13] A. Naaim, F. Moissenet, S. Duprey, M. Begon, and L. Cheze, (2017, Jan.). Effect of various upper limb multibody models on soft tissue artefact correction: A case study. J Biomech.

[14] A. Naaim, F. Moissenet, R. Dumas, M. Begon, and L. Cheze, "The neglected degree of freedom methods: a new method for defining marker set in motion analysis," in 22nd Congress of the European Society of Biomechanics, Lyon, France, 2016.

[15] M. Begon, C. Belaise, A. Naaim, A. Lundberg, and L. Cheze, (In Press, Oct.). Multibody kinematics optimization with marker projection improves the accuracy of the humerus rotational kinematics. J Biomech.

[16] W. Wu, P. V. Lee, and D. C. Ackland, (2017, Mar.). The sensitivity of shoulder muscle and joint force predictions to changes in joint kinematics: A Monte-Carlo analysis. Gait Posture. 54(87-92.

[17] F. Dal Maso, M. Raison, A. Lundberg, A. Arndt, and M. Begon, (2014, Nov.). Coupling between 3D displacements and rotations at the glenohumeral joint during dynamic tasks in healthy participants. Clin Biomech. 29(9), pp. 1048-1055.

[18] F. C. van der Helm, (1994, May.). A finite element musculoskeletal model of the shoulder mechanism. J Biomech. 27(5), pp. 551-569.

[19] B. Michaud, M. Jackson, A. Arndt, A. Lundberg, and M. Begon, (2016, Mar.). Determining in vivo sternoclavicular, acromioclavicular and glenohumeral joint centre locations from skin markers, CT-scans and intracortical pins: A comparison study. Med Eng Phys. 38(3), pp. 290-296.

[20] M. Begon, M. S. Andersen, and R. Dumas, (Submitted, .). Multibody kinematic optimization for the estimation of upper and lower limb human joint kinematics: a systematic review. J Biomech.

[21] C. R. Dickerson, D. B. Chaffin, and R. E. Hughes, (2007, Dec.). A mathematical musculoskeletal shoulder model for proactive ergonomic analysis. Comput Methods Biomech Biomed Engin. 10(6), pp. 389-400.
[22] Y. Blache, T. Creveaux, R. Dumas, L. Cheze, and I. Rogowski, (2017, Sep.). Glenohumeral contact force during flat and topspin tennis forehand drives. Sports Biomech. 16(1), pp. 127-142.

[23] K. M. Steele, M. S. Demers, M. H. Schwartz, and S. L. Delp, (2012, Apr.). Compressive tibiofemoral force during crouch gait. Gait Posture. 35(4), pp. 556-560.

[24] G. Bergmann, F. Graichen, A. Bender, A. Rohlmann, A. Halder, A. Beier, and P. Westerhoff, (2011, May.). In vivo gleno-humeral joint loads during forward flexion and abduction. J Biomech. 44(8), pp. 1543-1552.

[25] A. A. Nikooyan, H. E. Veeger, P. Westerhoff, F. Graichen, G. Bergmann, and F. C. van der Helm, (2010, Nov.). Validation of the Delft Shoulder and Elbow Model using in-vivo glenohumeral joint contact forces. J Biomech. 43(15), pp. 3007-3014.

[26] A. A. Nikooyan, H. E. Veeger, E. K. Chadwick, M. Praagman, and F. C. Helm, (2011, Dec.). Development of a comprehensive musculoskeletal model of the shoulder and elbow. Med Biol Eng Comput. 49(12), pp. 1425-1435.

[27] M. Laitenberger, M. Raison, D. Delphine Périé, and M. Begon, (2015, May.). Refinement of the upper limb joint kinematics and dynamics using a subject-specific closed-loop forearm model. MultiBody Syst Dyn. 33(4), pp. 413-438.

[28] V. Fohanno, M. Begon, P. Lacouture, and F. Colloud, (2013, Apr.). Estimating joint kinematics of a whole body chain model with closed-loop constraints. Multibody System Dynamics. 31(4), pp. 433449.

[29] J. L. Hicks, T. K. Uchida, A. Seth, A. Rajagopal, and S. L. Delp, (2015, Feb.). Is my model good enough? Best practices for verification and validation of musculoskeletal models and simulations of movement. J Biomech Eng. 137(2), pp. 020905.

[30] D. F. Massimini, P. J. Boyer, R. Papannagari, T. J. Gill, J. P. Warner, and G. Li, (2012, Jun.). In-vivo glenohumeral translation and ligament elongation during abduction and abduction with internal and external rotation. J Orthop Surg Res. 7(29), pp. 1:9.

[31] G. Lamberto, S. Martelli, A. Cappozzo, and C. Mazza, (In Press, Aug.). To what extent is joint and muscle mechanics predicted by musculoskeletal models sensitive to soft tissue artefacts? J Biomech.

[32] C. A. Myers, P. J. Laz, K. B. Shelburne, and B. S. Davidson, (2015, May.). A probabilistic approach to quantify the impact of uncertainty propagation in musculoskeletal simulations. Ann Biomed Eng. 43(5), pp. 1098-1111.

[33] Y. Blache, L. Desmoulins, P. Allard, A. Plamondon, and M. Begon, (2015, Nov.). Effects of height and load weight on shoulder muscle work during overhead lifting task. Ergonomics. 58(5), pp. 748-761.

[34] D. Gagnon, A. C. Babineau, A. Champagne, G. Desroches, and R. Aissaoui, $(2015$, .). Trunk and shoulder kinematic and kinetic and electromyographic adaptations to slope increase during motorized treadmill propulsion among manual wheelchair users with a spinal cord injury. Biomed Res Int. 2015, pp. 636319.

[35] S. van Drongelen, L. H. van der Woude, and H. E. Veeger, (2011, Jun.). Load on the shoulder complex during wheelchair propulsion and weight relief lifting. Clin Biomech. 26(5), pp. 452-457.

[36] X. Robert-Lachaine, P. Allard, V. Godbout, P. Tetreault, and M. Begon, (2016, Oct.). Scapulohumeral rhythm relative to active range of motion in patients with symptomatic rotator cuff tears. $J$ Shoulder Elbow Surg. 25(10), pp. 1616-1622.

[37] K. R. Holzbaur, W. M. Murray, and S. L. Delp, (2005, Jun.). A model of the upper extremity for simulating musculoskeletal surgery and analyzing neuromuscular control. Ann Biomed Eng. 33(6), pp. 829-840.

[38] T. Steele, A. Merryweather, C. R. Dickerson, and D. Bloswick, $(2013$, .). A computational study of shoulder muscle forces during pushing tasks. Int. J. Human Factors Modelling and Simulation. 4(1), pp. 1-22. 